\title{
Herr Frühling und Frau Winter
}

Authors: $\quad$ Mitra Zarif-Kayvan

Submitted: $\quad$ 6. December 2017

Published: $\quad$ 8. December 2017

Volume: 4

Issue: 6

Languages: German, Persian

Keywords: $\quad$ Persian literature; Fairy tale; Spring; Winter; change of seasons

DOI: $\quad$ 10.17160/josha.4.6.368

\section{JOSHA Jomanatosionece, Humanities and Arts}


عمو نوروز وننه سرما : ع مئ :

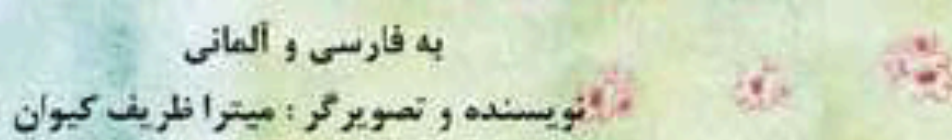

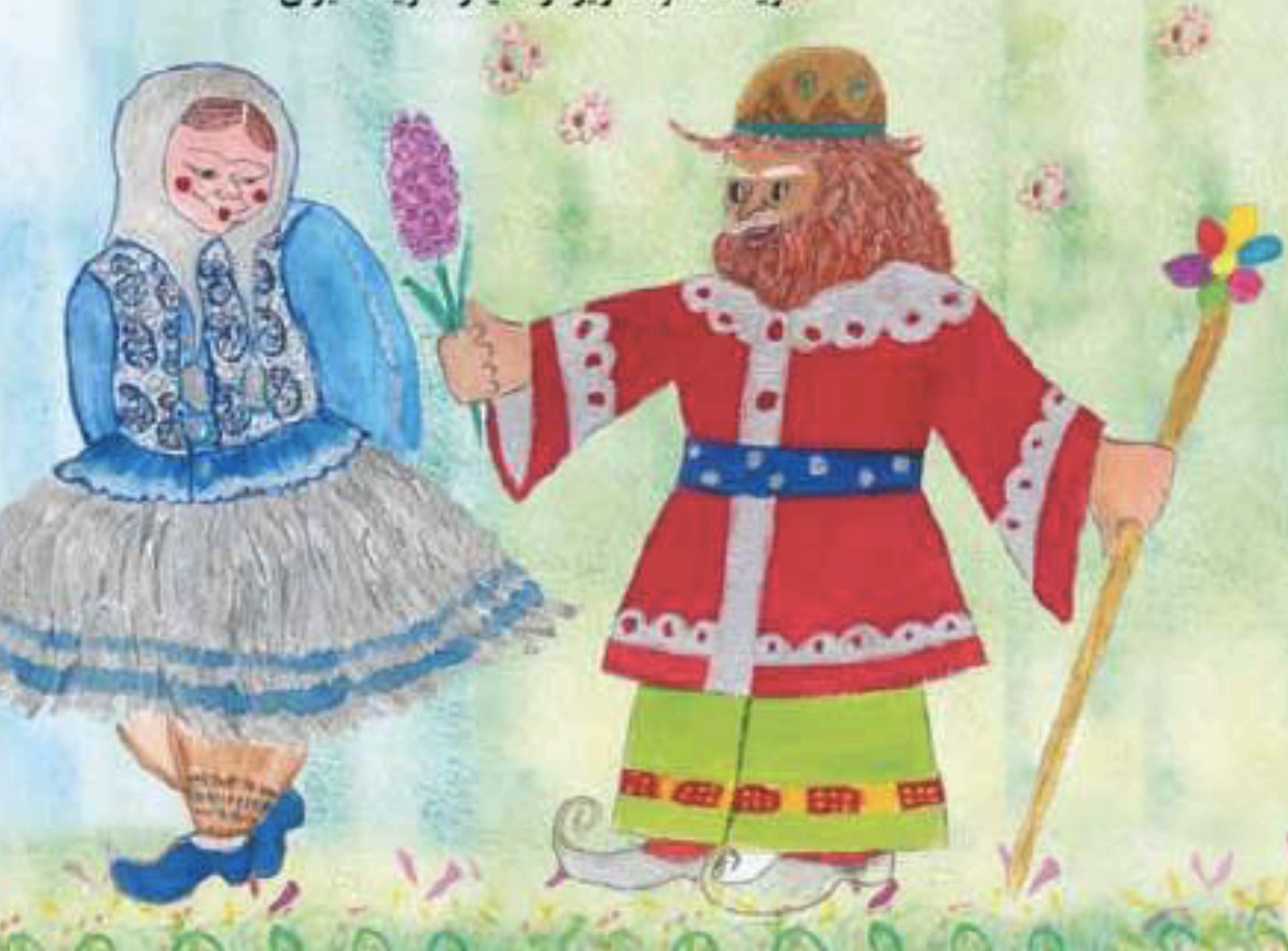

\section{Herr Frühling und Frau Winter} In Farsl und Doutsch

Autorin und Illustratorin Mitra Zarif-Kayvan

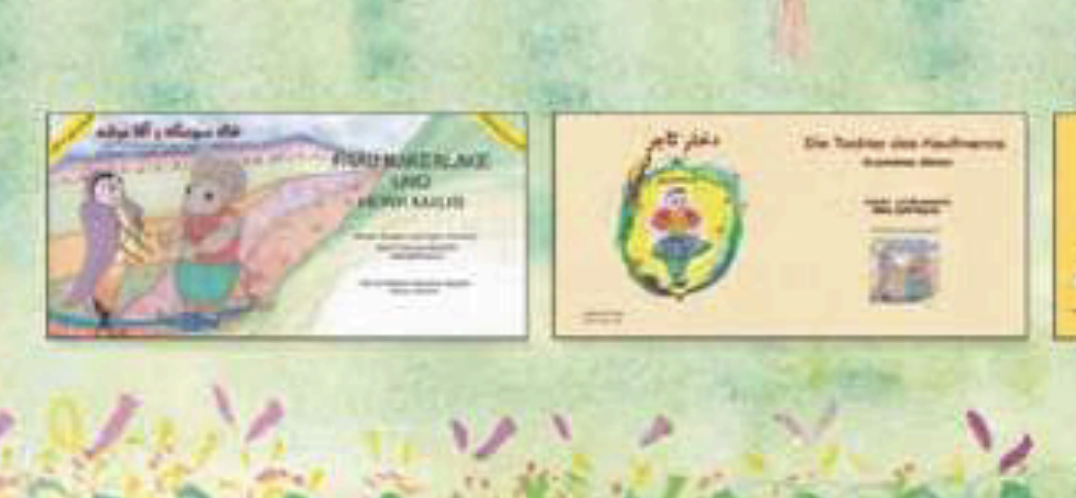

ار الئ نويستده عقتشر شده است:

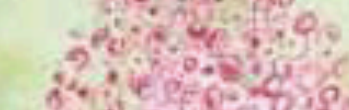

esoris

O.,

iforising

tet $22.1 \%$

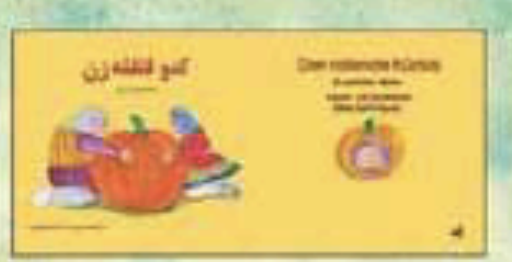

Wio of $Q$ 


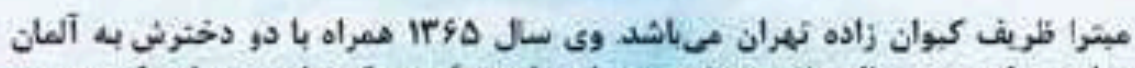

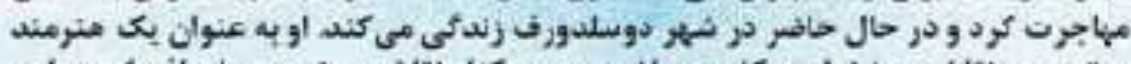

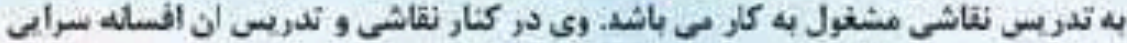

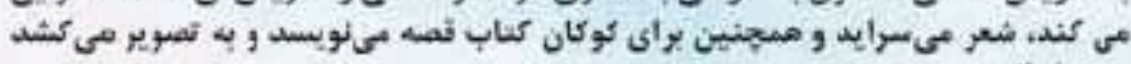

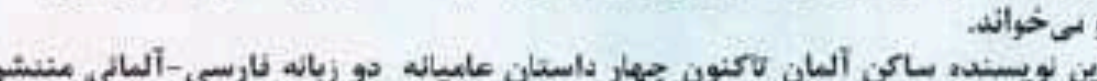

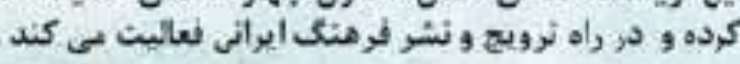

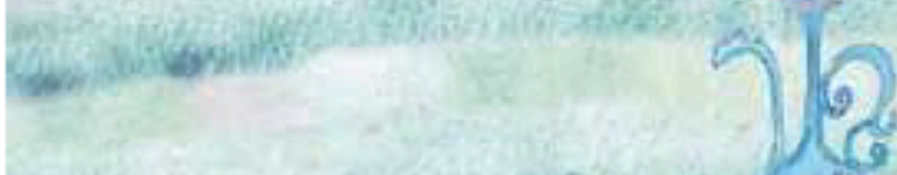

Zur Autorir und Illustration:

Mitra Zarif-Kayvan wurde in Teheran/Iran geboren. 1986 wanderte sie mit ihren beiden Töchtern nach Deutschland aus und lebt heute in Düsseldorf. Sie arbeitet als freischaffende Kuastmalerin und Kunstlehrerin. Daneben tritt sie als Mărchererzählerin auf, schreibt Gedichte und Erzählungen, illustriert, schreibt und liest Bilderbüeher für Kindet. Dieses Bilderbuch ist ihre vierte interkulturtle Arbeit in diesem Bereich.

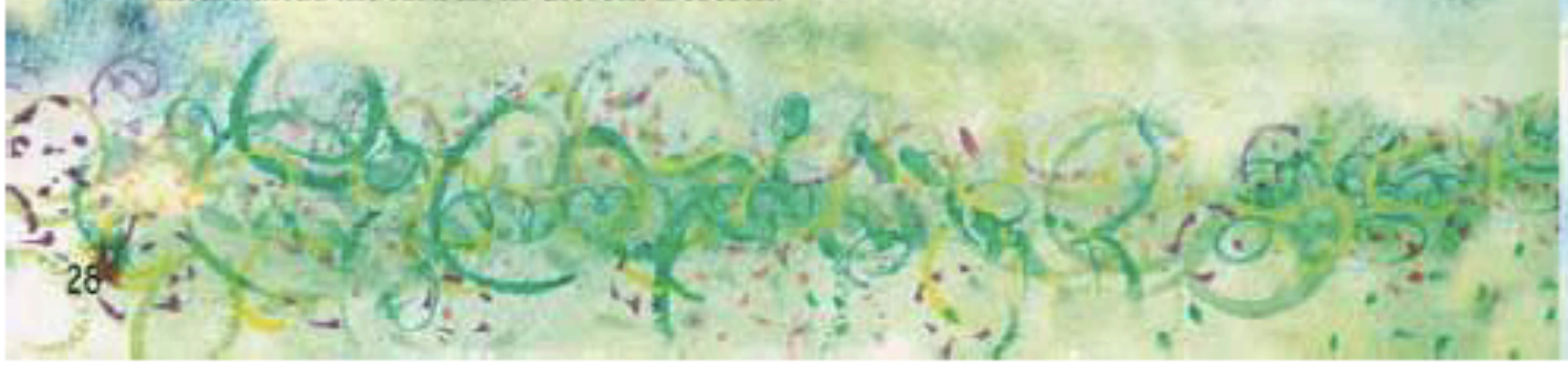

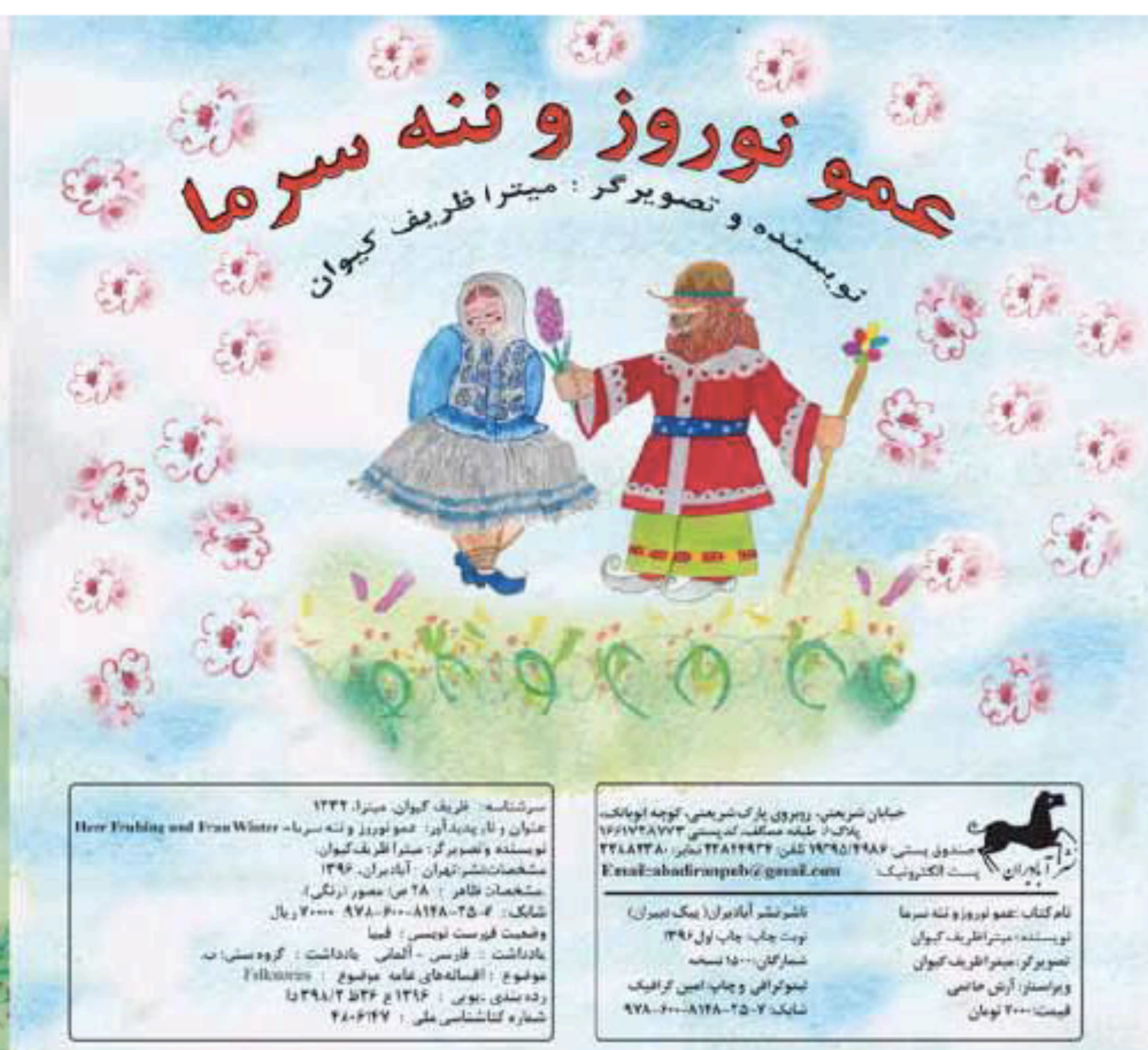


Die persische Literatur ist seit ch und je ęeprìgt durch Erzihlungen, Sagen. Sprichwörtern, Kurz- und Langgeschichten tiber die Veränderungen in allen Lebenslagen der Menschteit und den Veranderungan der Natur durch den Wechsel der Jahreszeiten.

Viele Malrehen wurden von ciner Generation in die rŭchste weitergetragen and erhielten bis heute eine andere und attuelle Ferm.

Das Mãrchen über die Liche zwischen Herm Frühlitg (Amou Noroux) und Frau Winte

(Nanne Sarma) ist schätzungsweise éber 2000 Jahre alt.

In dieser alten Erzăhlung symbolisiert Herr Frühling den Neubeginn, das Wiedererwadhen und

Wachstuan der Natur und das perisehe Neujahrsfest (Norouz) nit Bcginn des Frâhling.

Im Gegensatz daze stell Frau Winter Frost, Starre end stillstand dar.

Beide Charaktere spiezeln im Zusammenspiel das Eade des persisehen Jahres mit dem Ende des

Winters und dea Beghinn des neven Jahres mit dew fröhlingsanfang.

In einem Augenbilick beendet r rau Winter mit ihrem Abyang die Kälte und im gleichen Moment bringt Herr Frillalng mit stiner Ankunft die ersten Vorbeten des Fröblings.

Beide hätten aur aine einzige kurzweilige Gelegenhek einer gemeinsamen Begegnung

Mit freundlicher Vnterstützung ven Frau Ghazalch Hiazen (Shzyan) und Herrn Jan Michaclis beim deutschen Toxi.

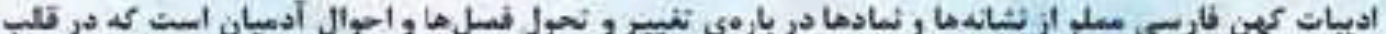

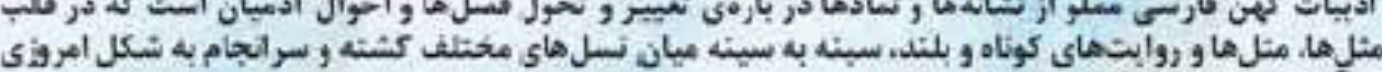

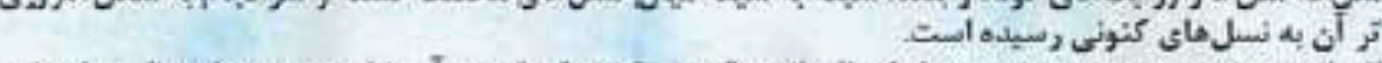

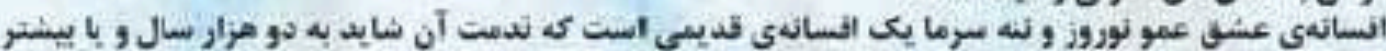

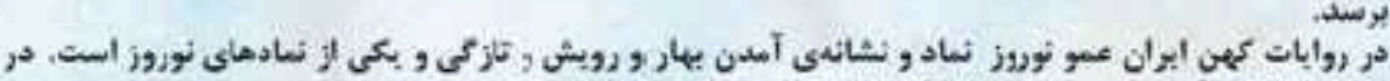

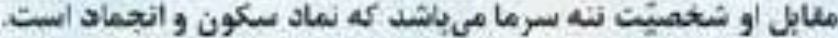

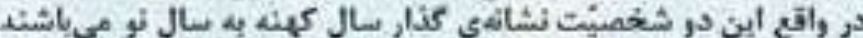

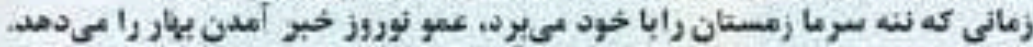

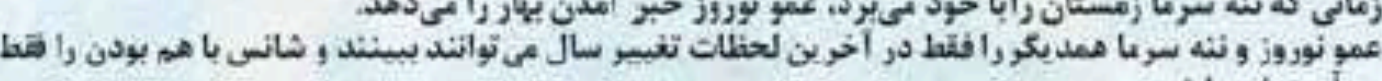

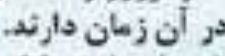
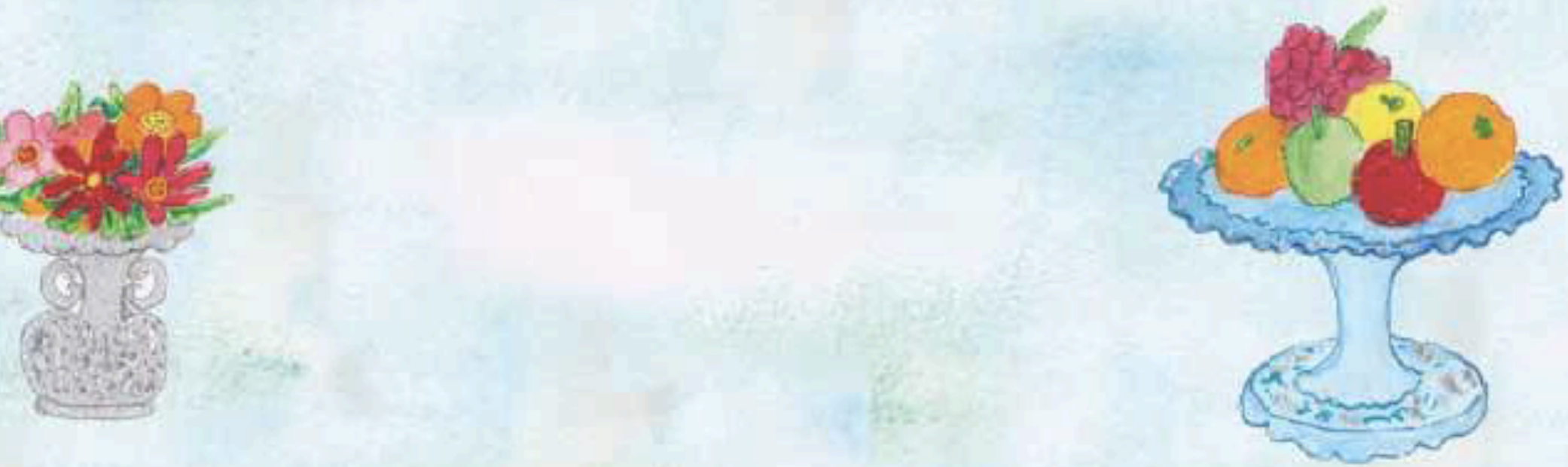


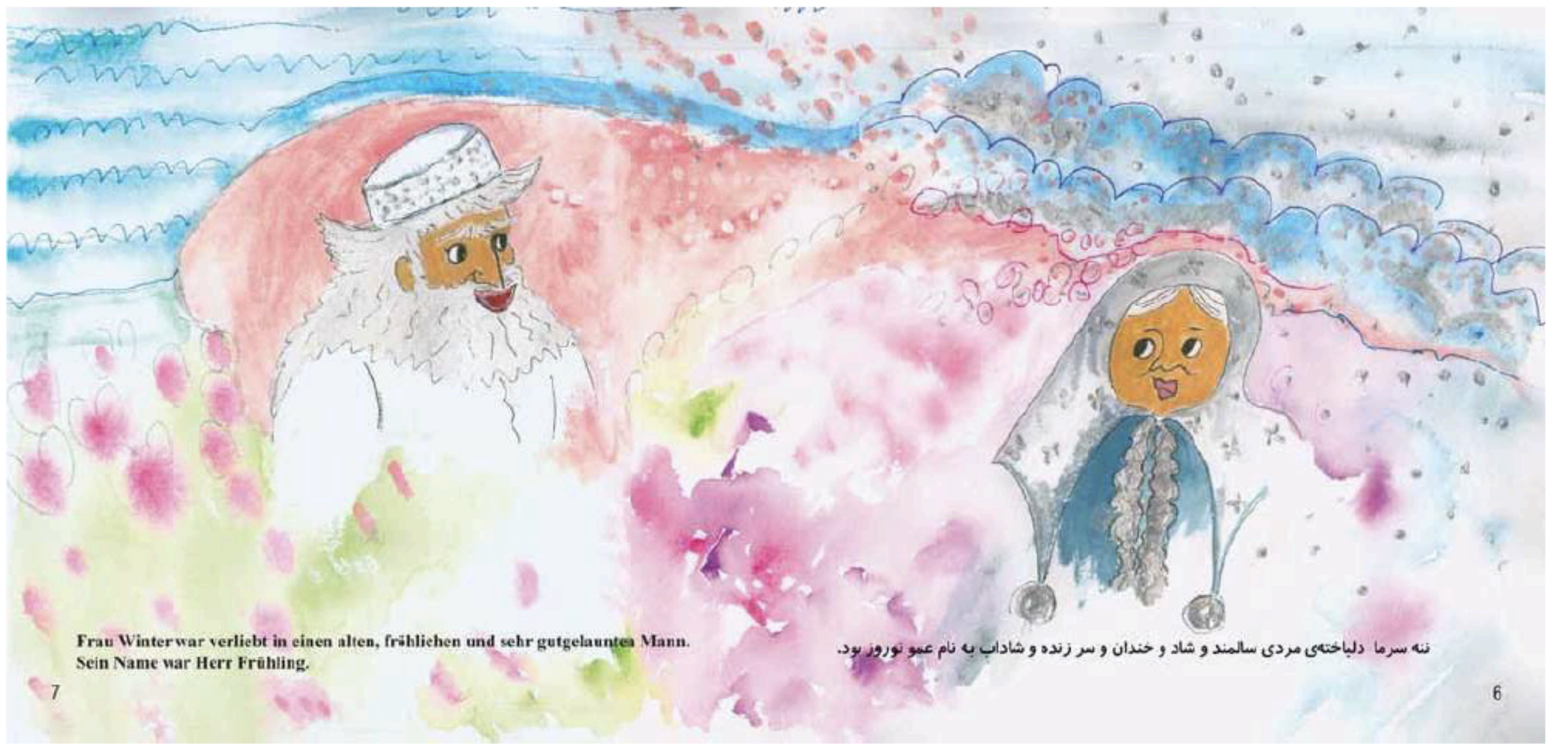

